\title{
Assessment of Power Level Variations in a Small PV System
}

\author{
P. Janik ${ }^{2}$, Z. Waclawek ${ }^{1}$, J. Rezmer ${ }^{1}$ \\ ${ }^{1}$ Department of Electrical Engineering \\ Wroclaw University of Technology \\ Wyb. Wyspianskiego 27, 50-370 Wroclaw (Poland) \\ Phone number:+48713202901, e-mail: przemyslaw.janik@ pwr.edu.pl \\ ${ }^{2}$ Faculty of Mechanical, Electrical and Industrial Engineering \\ Brandenburg University of Technology \\ Siemens-Halske-Ring 13, 03046 Cottbus (Germany) \\ Phone/Fax number: +49 355 694226, e-mail: przemyslaw.janik@tu-cottbus.de
}

\begin{abstract}
There is a significant content of high variations of power levels in a PV system. This variations are considered as an deterioration of power quality and therefore should be quantified, further minimised. The ripple content in the power curve was assessed with wavelets. A bell shaped function was correlated to a power curve using optimisation techniques. It resulted in a very compact characterisation of the power curve and enabled conceptual correlation between real power curve and power output line required by the utility.
\end{abstract}

\section{Key words}

PV systems, wavelet transform, active power, fluctuations

\section{Introduction}

A small PV installation mounted on the roof of a single family house is one of the most favourite solutions among prosumers. It is relatively simple to install and maintain. Some installation rules [1] and standards, i.e. [2]. give a clear guidance for the setting of PV installations. Also legal regulations simplify the connection to the public grid and the trade with electrical energy from small PV installations.

A developing trend in architecture is the use of building integrated photovoltaic. The panels are not more a spate object optimised for energy gain, but their design and layout is focused on aesthetic aspects.

PV generation is characterised by short and long term variations (seasonal, daily, instantaneous) which are immanent and contradictory to an desired ability to follow a predefined generation profile. New methods for the characterisation of power generation profiles, description of variations and distortion are needed. These characterisation and corresponding indices are not only significant form the engineering point of view, but give a tool for flexible energy price calculation accordingly to new power quality features.

\section{Research PV installation}

\section{A. PV System Rated $15 \mathrm{~kW}$}

The research installation with maximal power output 15 $\mathrm{kW}$ consists of three independent one phase systems constructed in different technologies. Schematic view is shown in Fig. 1. The photovoltaic research installation is located at the university campus and arranged on the roof of an existing building. The geographical orientation of the roof was accepted and no movable mounting structures were used.

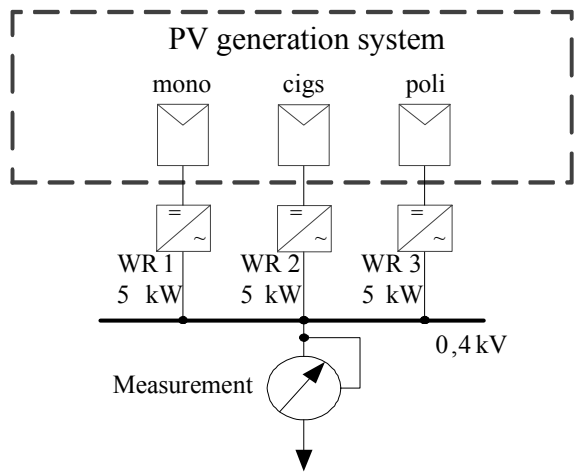

Fig. 1. Schematic view of $15 \mathrm{~kW}$ PV installation

\section{B. PV System Rated $110 \mathrm{~kW}$}

The photovoltaic installation consists of 530 modules with each $220 \mathrm{~W}_{\text {peak }}$, therefore $116.6 \mathrm{~kW}_{\text {peak }}$ for the whole generation facility. The modules of the company Algatec have a nominal voltage of $27.22 \mathrm{~V}$, a nominal current of $8.22 \mathrm{~A}$ and a modul-efficiency of $14.9 \%$. The PV panels are distributed over the main roof, the projecting roof and the facade (368 modules with an angle of incidence of $30^{\circ}, 162$ modules with an angle of incidence of $70^{\circ}$; the building is oriented $12^{\circ}$ to the south-west). The installation's currents and voltages are registered every second and can be retrieved anytime for evaluation and analysis. 
530 polycrystalline PV-Modules seperated to 7 strings

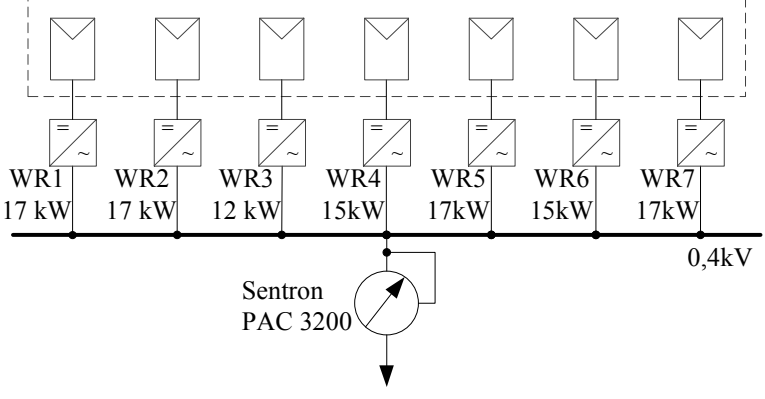

Fig. 2. Schematic view of $110 \mathrm{~kW}$ PV installation

\section{Daily Power Curves}

\section{A. Power Variations}

The daily power output of a PV installation is highly unstable due to seasonal changes and weather conditions variations in cloud coverage or temperature. Transients in solar irradiation are directly determining the shape of the power curve. Fig. 3 shows the active power output from the $110 \mathrm{~kW}$ installation on three subsequent days in July. Not only the days differ significantly from each other but also the variations during a day are noticeable. The changes correlate well with irradiation curves. Active power values were averaged over the period of one second in Fig. 3.

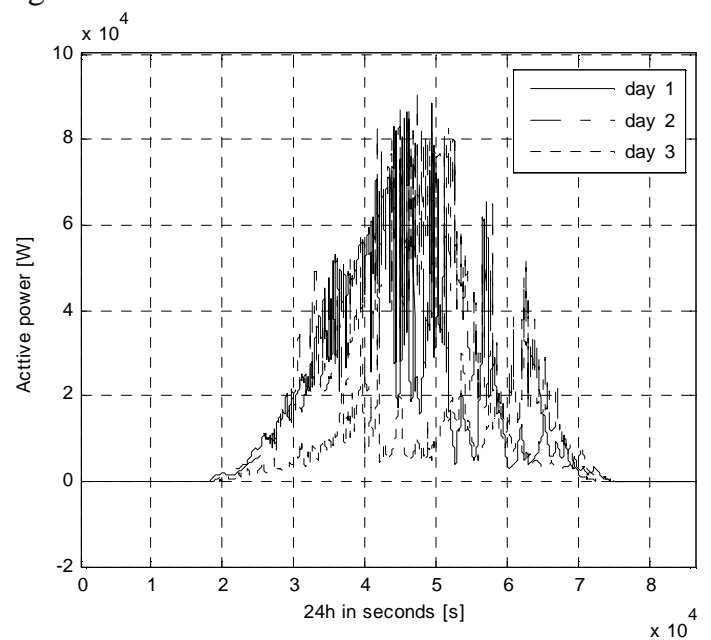

Fig. 3. Active Power on three subsequent days in July.

\section{B. Averaging}

There is an open question for the proper time of averaging. Utilities often propose $15 \mathrm{~min}$. values for power measurement. However, this time may be 10 min. or less if requested and justified [4]. Many power quality indices should be reported every $10 \mathrm{~min}$. [5]. Smart meters [6] enable measurement and data transfer every one minute or even less. Increase in sampling frequency has some disadvantages. Short measurement window reduces the number of smart meters which can be served by one concentrator and increases the amount of data to be stored and evaluated. Continuous measuring of active power values averaged over 200 milliseconds window (as for some power quality analysis) is relatively fare going proposal regarding data amounts and data transfer capabilities in public distribution grids.
Preliminary research results showed that data acquisition every one minute is satisfactory in the approaches presented later on. On the one hand, the data amount to be stored and analysed every day is reasonable and not voluminous. On the other hand, computational results are more precise and accurate than for data averaged over longer periods, $10 \mathrm{~min}$., or $15 \mathrm{~min}$. Therefore, one minute averaging period was selected and used in further analysis.

Data acquisition intervals used in both PV systems were different. To bring all data to the aforementioned one minute interval a procedure called resampling was applied [7], [8].

Daily power curve acquired with 1 second averaging period is shown in Fig. 4. Its counterpart after downsampling with 1440 samples per 24 hours (one minute period) is given in Fig. 5. Small dots in Fig. 5 indicate exact values.

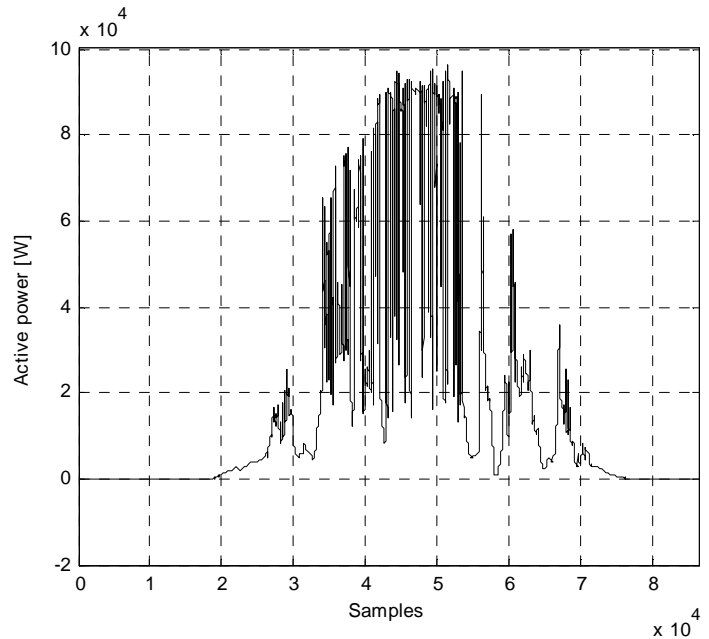

Fig. 4. Active Power, one second resolution, before resampling

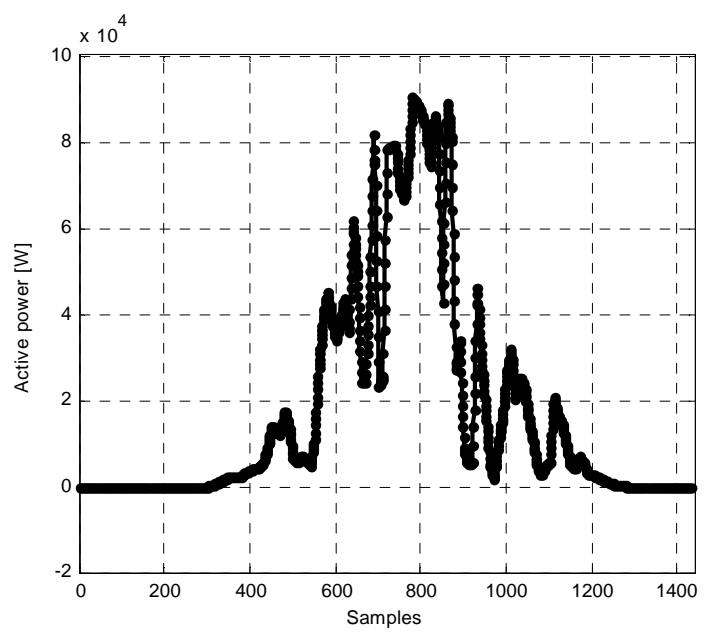

Fig. 5. Active Power, one minute resolution, after resampling

\section{Computation of centroids}

One of the simplest methods giving only two descriptive points a day is a centroid. It indicates the mass centre of the area below the plot as in Fig. 6. Generally, the horizontal and vertical coordinates are [3]

$$
x_{c}=\frac{1}{\text { Area }} \int_{a}^{b} x(f(x)-g(x)) d x
$$




$$
y_{c}=\frac{1}{\text { Area }} \int_{a}^{b}\left(\frac{f(x)-g(x)}{2}\right)(f(x)-g(x)) d x
$$

where

$$
\text { Area }=\int_{a}^{b}(f(x)-g(x)) d x
$$

Fig.6 indicates additionally the mean value as another simple descriptive feature of a PV power curve.

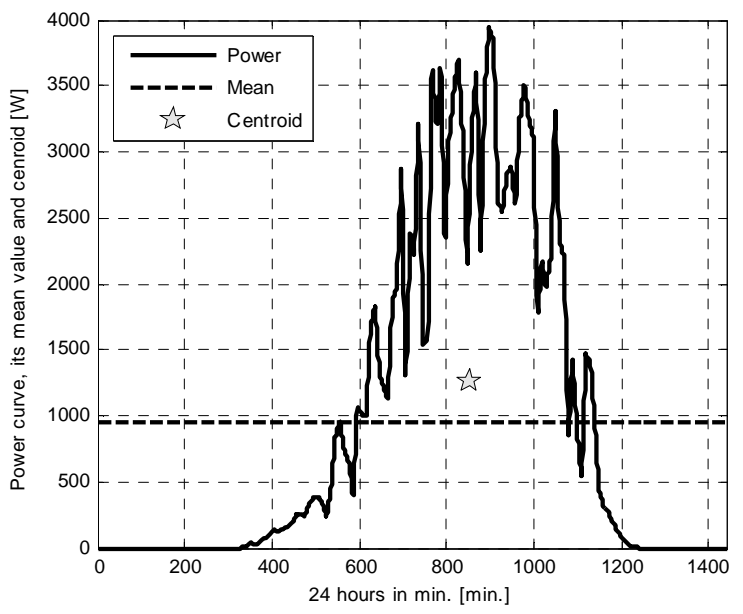

Fig. 6. Active power curve characterised by its centroid and mean value

\section{Approximation with a predefined shape}

Further description possibilities include a similarity identification of the power line shape to a predefined curve. It is advantageous, if power production in a PV system is coherent with a predefined function, e.g.:

- a constant line (possible with storage)

- covering a preselected demand curve (possible with storage)

- bell shape (possible with storage)

Without implementation of storage it is possible to find the parameters of a curve that best suits a predefined shape type given by a parametric equation, e.g. (4).

The aberration from predefined curve can be seen as parameter for the production pattern description and possible fees included in tariff regulations. In a statistical sense it can also be used or the sizing of a storage device allowing strict following of that curve.

The parameters of a predefined curve, fitting optimally the daily production are an index describing the curve in short and therefor predestined to be a quality factor.

For the energy production curve approximation an exponential bell function was used in this work. The general expression for this bell function is

$$
y_{\text {comp }}(t)=\alpha_{1} e^{-\left(\alpha_{2}\left(t-\alpha_{3}\right)\right)^{2}}
$$

where

$\alpha_{1}, \alpha_{2}, \alpha_{3}$ are descriptive parameters. Those parameters are determined in an optimisation process minimising the squared error between real power curve $y(\mathrm{t})$ and the bell shape

$$
y_{\text {error }}=\left\|\mathrm{y}(\mathrm{t})-\mathrm{y}_{\text {comp }}(\mathrm{t})\right\|
$$

Optimisation procedure was performed using a method based on evolutionary approach and using a classical gradient based iterative approach.

The plot of the power curve and its bell function approximation are shown in Fig. 7. Detailed values of parameters in (4) are given in Table I.

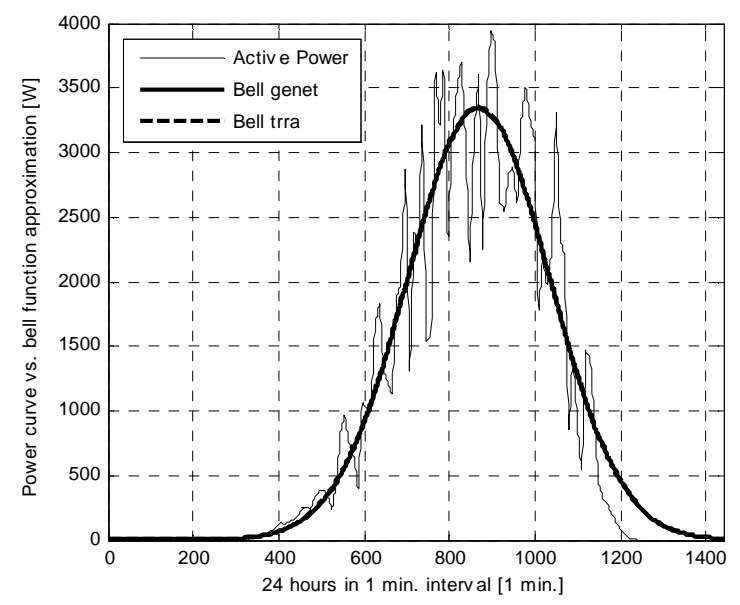

Fig. 7. Bell shape function approximating of daily power curve with distortions

Table I. - Bell function parameters

\begin{tabular}{|c|c|c|}
\hline parameter & genetic algorithm & $\begin{array}{c}\text { trust region refl. } \\
\text { algorithm }\end{array}$ \\
\hline$\alpha_{1}$ & 3214.388 & 3350.715 \\
\hline$\alpha_{2}$ & 0000.004 & 0000.004 \\
\hline$\alpha_{3}$ & 0870.956 & 0868.058 \\
\hline$y_{\text {error }}$ & 297780.626 & 289902.533 \\
\hline
\end{tabular}

The previous example contains a significant ripple. The next plot (Fig. 8) is more smooth in nature. Active power ant its bell function approximation are shown respectively.

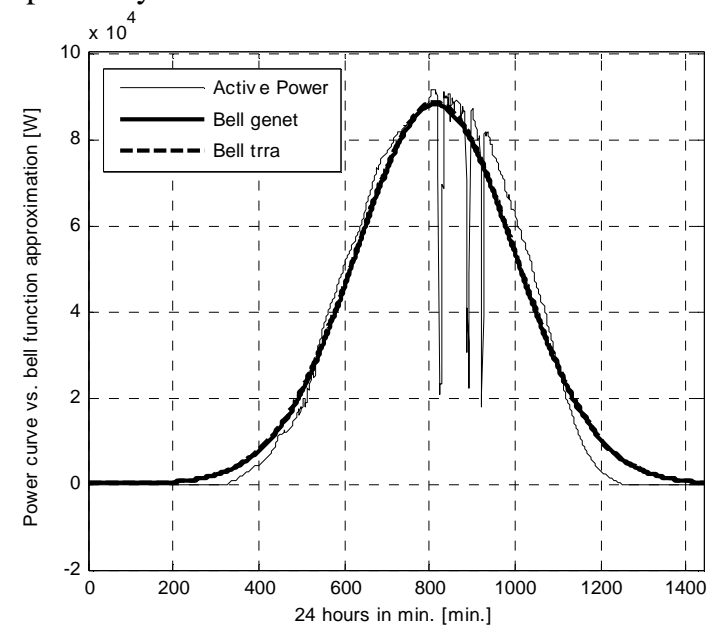

Fig. 8. Bell shape function approximating of smooth daily power curve

The detailed values of approximation parameters with accordance to (4) are given in Table II.

Table II. - Bell function parameters

\begin{tabular}{|c|c|c|}
\hline parameter & genetic algorithm & $\begin{array}{c}\text { trust region refl. } \\
\text { algorithm }\end{array}$ \\
\hline$\alpha_{1}$ & 88474.797 & 88566.751 \\
\hline$\alpha_{2}$ & 0000.038 & 0000.038 \\
\hline$\alpha_{3}$ & 0081.839 & 0081.839 \\
\hline $\mathrm{y}_{\text {error }}$ & 538274.829 & 537600.676 \\
\hline
\end{tabular}




\section{Wavelet Transform}

The power output of a PV power generation unit is highly variable, as previously indicated.

The daily irradiation and power curves overlap (ignoring different axes scaling) and correspond to each other. Multiplication of the irradiation by an coefficient enables a true match between these two quantities (Fig. 9).

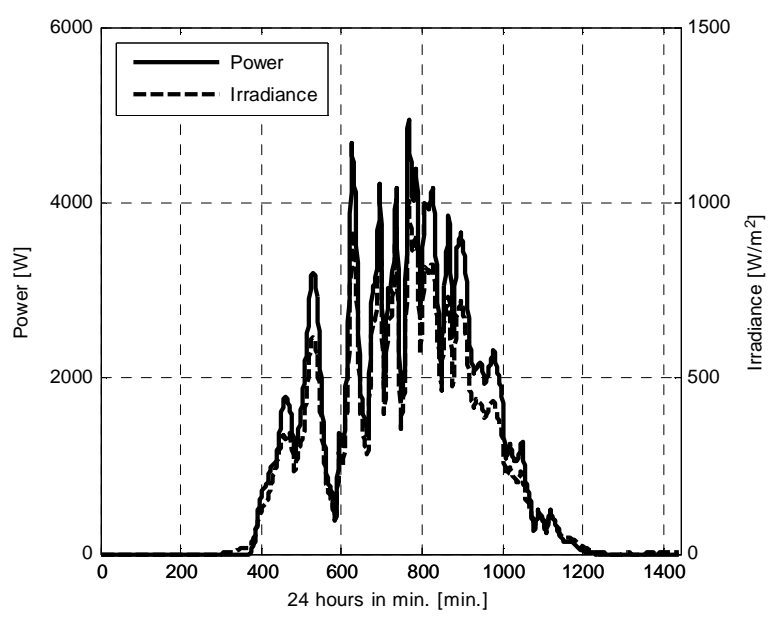

Fig. 9. Active power vs. irradiance

During the preliminary research various wavelet functions with varying parameters were used to assess the ripple content in daily power curves.

The function symlets [9] generates the least-asmmetric Daubechies' filters. This function returns the coefficients of the orthogonal finite impulse response filters with zeroes of the trigonometrical polynomial selected alternatively inside and outside the unit circle. Such a selection results in nearest design to linear phase and least asymmetric wavelets. As in daub the number of filter coefficients must be even.

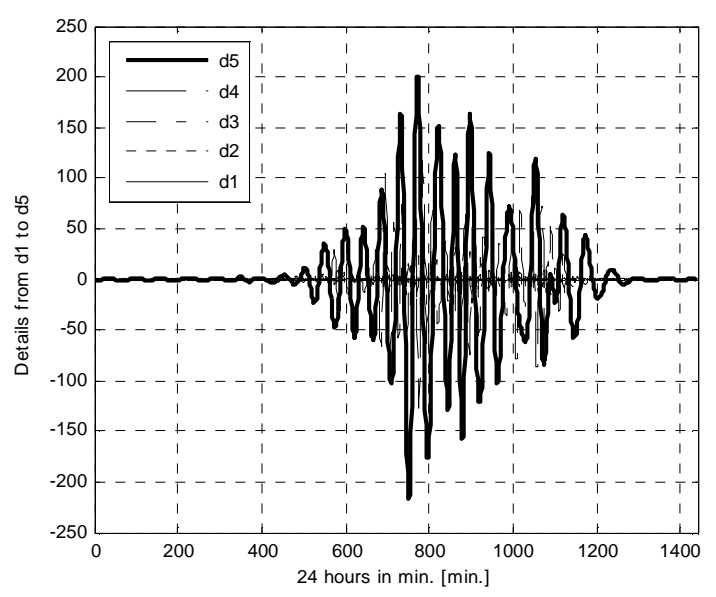

Fig. 10. The fifth detail of the daily power curve

Fig. 10 shows the first five details of a daily power curve on the same axes scale to enable a visual comparison between details. The particular detail no. 5 was plotted with a bold line. The highest number of a detail correspond to the lowest frequency content, the lowest number, i.e. one, indicates highest frequencies. Every higher level imposes the frequency range division of the preceding approximation (original signal in the first step) by two.
The proposed description method for variations in a daily power curve is based on the computation of details. Parameters as min./max. value of the detail, integral of the detail over time, are usefully descriptive and informative. Additionally, the basic ideas of descriptive statistics were used [10].

Table III. - Descriptive parameters of details

\begin{tabular}{|c|l|l|l|}
\hline detail no. & min. & max. & integral \\
\hline d1 & -046.311 & 0049.294 & 2604.664 \\
\hline d2 & -075.638 & 0072.750 & 8627.303 \\
\hline d3 & -185.539 & 0151.170 & 21589.907 \\
\hline d4 & -486.890 & 0528.708 & 75670.797 \\
\hline d5 & -817.111 & 0732.435 & 165429.156 \\
\hline
\end{tabular}

\section{Summary}

The aim of the work was assessment of daily power curve ripples common in PV installations.

A bell function approximation was proposed along with a genetic algorithm based parameter estimation. Despite high fidelity of both methods and very similar parameters of the daily power curve approximations it seems advantageous to suggest evolutionary based algorithm for further practical applications.

Wavelet transform is regarded to be an appropriate tool for the analysis of daily power curves in PV installations. The approximation component describes the smooth curve, showing the low frequency block of the signal. The details include information about severity of changes happening in the signal. This information is included in the shape and index of the detail.

\section{References}

[1] VDE-AR-N 4105:2001-8, Power generation systems connected to the low voltage distribution network - technical minimum requirements for the connection and parallel operation with low voltage distribution networks, 2011

[2] EN 50438 Standard. Requirements for the connection of micro-generators in parallel with public low-voltage distribution networks, 2014

[3] R. E. Larson, R. P. Hostetler i B. H. Edwards, Calculus of a Single Variable, Boston: Houghton Mifflin Company, 1998

[4] Transmission Code 2007: Network and System Rules of the German Transmission System Operators. VDN, 2007

[5] PN-EN 50160 Standard. Voltage characteristics of electricity supplied by public electricity networks, 2010

[6] Q. Wells, Smart Grid Home, Clifton Park: Delmar, Cengage Learning, 2013

[7] R. G. Lyons, Understanding Digital Signal Processing, Reading: Addison Wesley, 2007.

[8] A. V. Oppenheim, A. S. Willsky and S. H. Nawab, Signals and Systems, Englewood Cliffs: Prentice Hall, 1997

[9] S. G. Sanchez, N. G. Prelcic i S. J. G. Galan, „Uvi Wave. Wavelt Toolbox for the use with Matlab," Universidad de Vigo, Vigo, 1996

[10] S. M. Ross, Introductory Statistics, Kidlington: Elsevier Inc., 2010 